\title{
Prevalencia de alteraciones cromosómicas en perros domésticos (Canis familiaris) con neoplasias: un estudio prospectivo
}

\author{
Prevalence of Chromosomal alterations in domestic dogs (Canis familiaris) \\ WITH NEOPLASMS: A PROSPECTIVE STUDY
}

\author{
Jeel Moya-Salazar ${ }^{1,2,4}$, Mario Verano-Zelada ${ }^{1}$, Rafael Vega-Vera $^{3}$
}

\section{Resumen}

\begin{abstract}
El presente estudio tuvo como objetivo determinar la prevalencia de las alteraciones cromosómicas en Canis familiaris con neoplasias clínicas. Se trabajó con 25 pacientes de una clínica veterinaria en Lima, Perú en 2015-2016. Para el cultivo de sangre periférica y el análisis cromosómico se colectaron muestras por punción venosa, las cuales se almacenaron por $3 \pm 1$ horas como máximo. Se realizó el procesamiento citogenético y el bandeo GTG convencional. El reporte se realizó según las recomendaciones del Committee for the Standardized Karyotype of the Dog. El promedio de edad fue de 9.5 años (4-17 años) y los grupos raciales con mayor frecuencia fueron el mestizo (20\%), Golden Retriever (16\%) y Shi-tzu (11.5\%). El 72\% presentaba alteraciones cromosómicas. De estas, $9(36 \%)$ fueron trisomías, $9(36 \%)$ heterocromatinas constitutivas, $2(8 \%)$ translocaciones, $2(8 \%)$ deleciones y 1 (4\%) cromosoma en anillo. Se observaron alteraciones mixtas en 9 pacientes $(36 \%)$, donde la trisomía del cromosoma 13 fue la más frecuente $(28 \%)$, seguida de heterocromatina del cromosoma 9 (24\%). El análisis de riesgo mediante regresión logística múltiple demostró que perros de 10 o más años tuvieron 2.4 veces más riesgo de cáncer que perros de menor edad $(\mathrm{p}<0.05)$.
\end{abstract}

Palabras clave: perros; análisis citogenético; neoplasias; prevalencia

\section{Abstract}

The objective of this study was to determine the prevalence of chromosomal alterations in Canis familiaris with clinical neoplasms. Twenty-five patients from a veterinary clinic in Lima, Peru in 2015-2016 were monitored. Blood samples were collected

\footnotetext{
${ }^{1}$ Departamento de Diagnóstico y Laboratorio Clínico, Laboratorio de Genética y Genómica Golberd Hognes Associated Veterinary and Oncology, Veterinaria Clini-Vet, Lima, Perú

${ }^{2}$ Facultad de Ciencias de la Salud, Universidad Continental, Huancayo, Perú

${ }^{3}$ Área de Citogenética Humana y Biología Molecular, Servicio de Anatomía Patológica, Hospital Nacional Docente Madre-Niño San Bartolomé, Lima, Perú

${ }^{4}$ Email: jeelms@outlook.com
}

Recibido: 3 de septiembre de 2017

Aceptado para publicación: 30 de enero de 2018 
by venous puncture for peripheral blood culture and chromosomal analysis and were stored for $3 \pm 1$ hours maximum. Cytogenetic processing and conventional GTG banding were performed. The report was made according to the recommendations of the Committee for the Standardized Karyotype of the Dog. The average age was 9.5 years (4-17 years) and the racial groups with greater frequency were the mestizo (20\%), Golden Retriever $(16 \%)$ and Shi-tzu (11.5\%). Chromosomal alterations were observed in $72 \%$ of the patients. Of these, $9(36 \%)$ were trisomies, $9(36 \%)$ constitutive heterochromatins, $2(8 \%)$ translocations, $2(8 \%)$ deletions and $1(4 \%)$ ring chromosome. Mixed alterations were observed in 9 patients $(36 \%)$, where the trisomy of chromosome 13 was the most frequent (28\%), followed by heterochromatin of chromosome 9 (24\%). Risk analysis using multiple logistic regression showed that dogs 10 years of age and older had 2.4 times more cancer risk than younger $\operatorname{dogs}(\mathrm{p}<0.05)$.

Key words: dogs; cytogenetic analysis; neoplasms; prevalence

\section{INTRODUCCIÓN}

El cáncer no solo se constituye en una de las principales causas de muerte en los seres humanos, sino que también afecta a diversas especies animales, entre las cuales se encuentra Canis familiaris (Dobson y Lascelles, 2014; Ujvari et al., 2017). Las neoplasias en animales poseen la misma tendencia que en seres humanos. Se calcula que uno de cada cuatro perros y gatos morirá de cáncer o de alguna enfermedad relacionada a esta patología (Vail y MacEwen, 2000; Dobson y Lascelles, 2014).

Las células cancerígenas poseen heterogeneidad genética que provee un pool de mutaciones que dirigen a que la progresión neoplásica mantenga la habilidad para evitar senescencia e impasividad, y la resistencia terapéutica que acelera la evolución del cáncer (Turner et al., 2017). La inestabilidad genética es uno de los factores requeridos para el desarrollo del cáncer (Hanahan y Weinberg, 2011), la que podría traducirse como mutaciones (deleciones, trisomías, traslocaciones y heterocromatinas, entre otras) (Maeda et al., 2012).
El uso de la citogenética resulta un elemento de mucha valía para el diagnóstico y seguimiento en neoplasias humanas linfoproliferativas como la leucemia y podría constituir en estos casos una herramienta de monitorización de estas afecciones en animales (Breen, 2008; Verano-Zelada et al., 2016). Del mismo modo, en perros se han documentado alteraciones del tipo trisomías libres del cromosoma 13 en linfomas, determinando que tienen mejor pronóstico en comparación con otros pacientes con linfoma (Verano-Zelada et al., 2016); sin embargo, la prevalencia de alteraciones cromosómicas en perros con procesos neoplásicos no ha sido reportada en el Perú.

El presente estudio estuvo destinado a reconocer, mediante el uso de técnicas en citogenética, las alteraciones cromosómicas en Canis lupus familiaris con neoplasias, así como su correlación con diversos determinantes de riesgo (supervivencia, edad, raza, gravedad de enfermedad), contribuyendo a un mejor entendimiento de esta condición en el paciente canino, y sus modificaciones en los arreglos cromosómicos, que podrían conducir una sobreexpresión de genes, por pérdida de la homeostasis celular entre proliferación y sus respectivos mecanismos de control. 


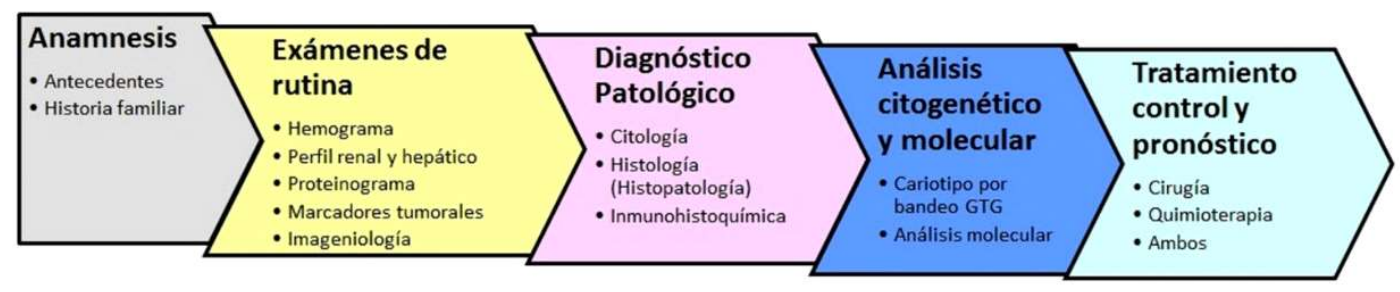

Figura 1. Flujograma de la atención sanitaria del paciente neoplásico. Los análisis en cada fase pueden variar dependiendo de la asistencia médica y la pertinencia clínica.

\section{Materiales y Métodos}

\section{Ubicación del Estudio y Pacientes}

Se realizó un estudio prospectivo de corte trasversal en el Laboratorio de Genética y Genómica «Golberd Hognes Associate Veterinary and Oncology » de la Clínica Veterinaria «Clini-Vet» en Lima, Perú, durante 2015 y 2016.

Se seleccionaron pacientes caninos con neoplasias de la unidad de oncología. A los pacientes se les realizó exámenes de rutina y complementarios como perfiles fisiológicos e imageneologia, entre otros, así como diagnósticos histopatológicos con el fin de establecer el estadio y grado de las neoplasias. El manejo de la patología fue mediante cirugía, quimioterapia o ambos. Todos los procedimientos se realizaron mediante la aprobación del Consentimiento Informado por parte del dueño con el fin de tener las herramientas necesarias para un mejor enfoque de la enfermedad y elegir el tratamiento apropiado que conduzca a mejorar la calidad de sobrevida del paciente (Figura 1).

\section{Muestras}

Se recolectó sangre periférica con jeringa de $5 \mathrm{ml}$ con $0.1 \mathrm{ml}$ de heparina sódica o con el sistema de extracción BD Vacutainer ${ }^{\mathrm{TM}}$ (Franklin Lakes, EEUU). Todas las muestras contuvieron $4 \pm 1 \mathrm{ml}$ de sangre venosa (se descartaron muestras con menos de $3 \mathrm{ml}$ o aparentemente contaminadas). Las muestras fueron procesadas frescas y en el menor tiempo posible, o almacenadas en refrigeración por $3 \pm 1 \mathrm{~h}$ como máximo.

\section{Análisis Citogenético}

Los cultivos de linfocitos de sangre periférica se realizaron en el medio KREAvital Lymphocyte Karyotyping (Kreatech Bio, Países Bajos) y en el medio PB-MAX ${ }^{\mathrm{TM}}$ Karyotyping (Thermo Fisher Scientific, EEUU) manteniéndose en incubación a $37 \pm 1{ }^{\circ} \mathrm{C}$ hasta por $96 \mathrm{~h}$ con controles de calidad de cultivo a las 48 y $72 \mathrm{~h}$ (Iannuzzi y Di Berardino, 2008).

Durante la cosecha se realizó la colchinización (con colcemid $0.1 \mu \mathrm{g} / \mathrm{ml}$ ), hipotonización con $\mathrm{KCl}$ a $0.075 \mathrm{M}$, y la prefijación y fijación con la solución de Carnoy comercial (Czepulkwski, 2001). Se utilizó el bandeo GTG con coloración Giemsa (modificado de Leishman's) (Seabright, 1971; Wang y Fedoroff, 1972).

Los extendidos microscópicos coloreados con Giemsa fueron analizados por microscopía de luz a 100X en el autoanalizador Leica System DFC340 FX (Leica, Alemania), con la finalidad de observar anormalidades cromosómicas de acuerdo con los ideogramas establecidos con patrones de bandas (Switonski et al., 1996, Verano-Zelada et al., 2016). El reporte de resultados se hizo mediante el sistema ISCN (International System for Cytogenetics Nomenclature) ar- 


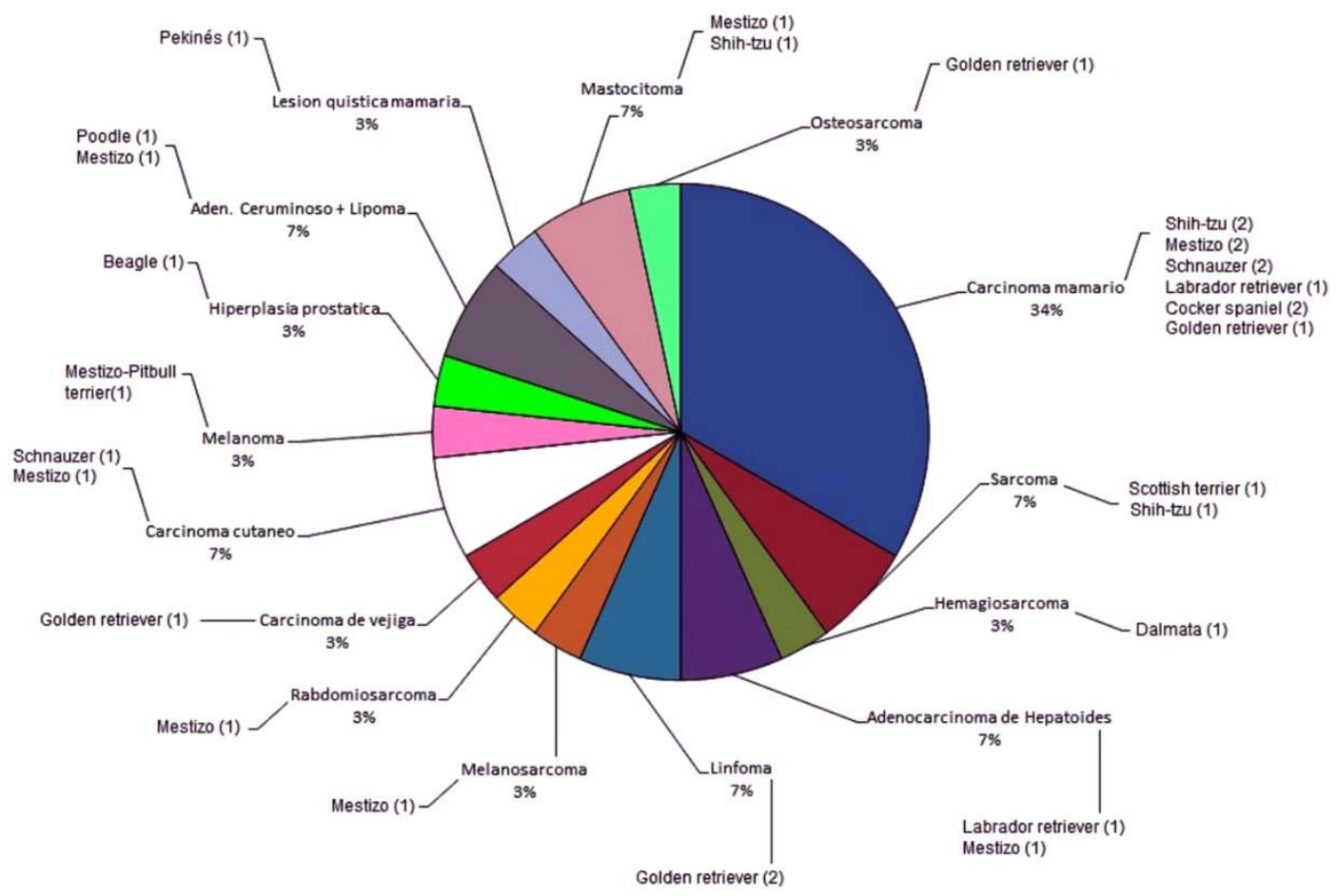

Figura 2. Distribución de neoplasias en perros domésticos con neoplasias $(\mathrm{n}=25)$ según la raza. Se han considerado neoplasias mixtas (las frecuencias de neoplasias se expresan en porcentaje, las frecuencias de razas en números cardinales)

ticulado para reportes citogenéticos de animales domésticos por el Committee for the Standardized Karyotype of the Dog en 1994 (Switonski et al., 1996).

\section{Análisis de Datos}

Los datos se obtuvieron del sistema de almacenamiento de datos de Leica System DFC340 FX (Leica, Alemania) para Windows. Se utilizó estadística descriptiva con base a frecuencias simples y promedios. Asimismo, se usó la correlación de Spearman para cada agrupación cromosómica alterada. El análisis de riesgo se realizó mediante regresión logística múltiple. Se consideró un valor de $\mathrm{p}<0.05$ como estadísticamente significativo. Los análisis estadísticos se realizaron con el programa IBM SPSS v. 21.0 para Windows.

\section{Resultados}

Se incluyeron 25 perros domésticos con diagnóstico patológico de neoplasia. El 37\% de perros presentó neoplasia mamaria. De la Figura 2 se infiere que el $20 \%(5 / 25)$ presentó neoplasias mixtas y el $4 \%(1 / 25)$ presentó neoplasia triple (adenocarcinoma, lipoma y mastocitoma).

El promedio de edad fue de 9.5 años (rango: 4-17 años). Se contó con 10 (40\%) pacientes machos y $15(60 \%)$ hembras, donde pacientes de ambos sexos fueron afectados en $\geq 70 \%$. No se encontró diferencia significativa entre la edad de los pacientes $(\mathrm{p}=0.778)$. Así mismo, las hembras presentaron trisomías en mayor cantidad que las heterocromatinas constitutivas como altera- 


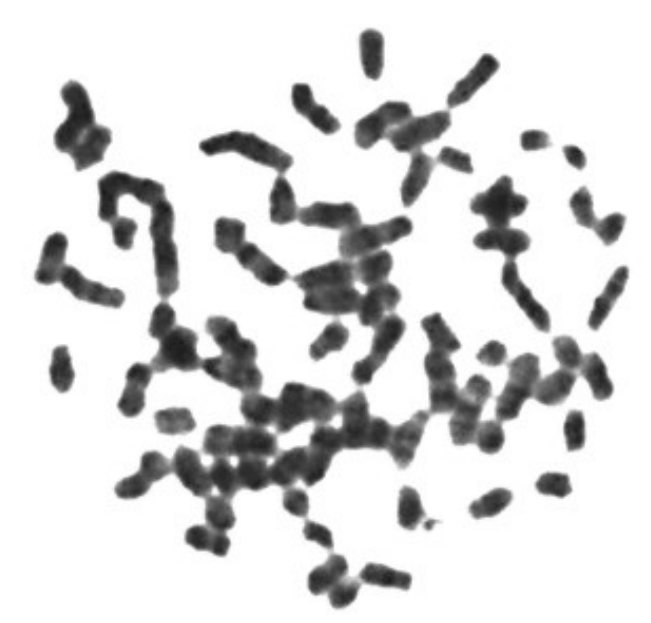

Figura 3. Paciente N. ${ }^{\circ} 03$ con linfoma cutáneo no B ni T, cariotipo 80, XX, $+13,+18$ (metafase celular con bandeo GTG)

ción citogenética de mayor frecuencia, mientras que en machos se encontró mayor número de heterocromatinas que trisomías. Se encontraron diferencias significativas entre los resultados del cariotipo y sexo $(\mathrm{p}=0.991)$, siendo más afectadas las hembras.

Trece $(68 \%)$ de los perros afectados fueron perros de raza de un total de 19 pacientes, en tanto que solo $5(83 \%)$ de los seis perros mestizos tuvieron alteraciones cromosómicas. Los grupos raciales con mayor frecuencia fueron mestizo (20\%) y Golden Retriever (16\%) (Cuadro 1). La prevalencia de alteraciones citogenéticas fue de $72 \%$ (18/25). Asimismo, 36\% (9/25) de los animales presentaron trisomías libres, 36\% (9/25) presentaron heterocromatinas constitutivas, $8 \%(2 / 25)$ tuvieron translocaciones, $8 \%(2 / 25)$ mostró deleciones y $4 \%(25 / 1)$ presentó cromosoma en anillo (Figura 3). Las alteraciones citogenéticas mixtas fueron $36 \%$ (9/25). Los cromosomas más afectados se detallan en el Cuadro 2. No se encontró diferencia significativa entre los resultados de cariotipo entre pacientes $(\mathrm{p}=0.510)$.

La mortalidad fue del 24\% (6/25) durante en el tiempo de estudio. Cinco pacientes hembras (tumor mamario) y un macho (tres tipos de tumores). Tres de los seis pa- cientes fallecidos tuvieron heterocromatinas constitutivas, dos tuvieron trisomías libres, dos tuvieron deleciones y solo uno presentó translocación. No se estableció una correlación directa entre la alteración cromosómica y la edad del paciente $(\mathrm{p}=0.544)$ ni con el sexo de los pacientes $(\mathrm{p}=0.955)$.

La neoplasia mamaria se constituyó en la de mayor mortalidad, seguido de linfoma, melanoma, y carcinoma de células transicionales de vejiga. Se encontró una relación directa significativa entre la mortalidad y la edad de los pacientes $(\mathrm{p}=0.018)$ y entre la mortalidad y el tipo de afección $(p=0.114)$, agravándose con el grado de alteración. El análisis de riesgo mediante regresión logística múltiple demostró que los perros $\geq 10$ años tuvieron 2.4 veces más riesgo de trastorno citogenético que los perros $<10$ años $(p<0.05)$.

\section{Discusión}

Se determinó una elevada prevalencia de alteraciones citogenéticas en perros domésticos con neoplasias, siendo las trisomías libres y las heterocromatinas constitutivas las de mayor cuantía. 
Cuadro 1. Resultados de alteraciones citogenéticas de 25 perros domésticos con neoplasias clínicas (Lima, Perú, 2015-2016)

\begin{tabular}{|c|c|c|c|c|c|}
\hline N. ${ }^{\circ}$ & Raza & $\begin{array}{l}\text { Edad } \\
\text { (años) }\end{array}$ & Neoplasia & Periodo & $\begin{array}{l}\text { Alteración } \\
\text { cromosómica }\end{array}$ \\
\hline 1 & Shi tzu & 7 & $\begin{array}{l}\text { Carcinoma mamario simple / } \\
\text { mastocitoma }\end{array}$ & $1 \mathrm{t}-2015$ & $79, \mathrm{XX},+13, \mathrm{r}(2)$ \\
\hline 2 & $\begin{array}{l}\text { Golden } \\
\text { Retriever }\end{array}$ & 6 & $\begin{array}{l}\text { Carcinoma células } \\
\text { transicionales de vejiga }\end{array}$ & $1 \mathrm{~T}-2015$ & $80, \mathrm{XX},+18,+24$ \\
\hline 3 & $\begin{array}{l}\text { Golden } \\
\text { Retriever }\end{array}$ & 6 & Linfoma cutáneo NO B ni T & $2 \mathrm{~T}-2015$ & $80, \mathrm{XX},+13,+18$ \\
\hline 4 & Beagle & 8 & Hiperplasia prostática & $2 \mathrm{t}-2105$ & $79, X Y,+13, t(10 ; 18)$ \\
\hline 5 & Pekines & 4 & Lesión quística mamaria & $2 \mathrm{~T}-2015$ & $78, \mathrm{XX}, 26 \mathrm{hq}+$ \\
\hline 6 & Shi tzu & 6 & Sarcoma oral bajo grado & $3 \mathrm{~T}-2015$ & $78, \mathrm{XY}$ \\
\hline 7 & Mestizo* & 17 & $\begin{array}{l}\text { Melanosarcoma/ } \\
\text { adenocarcinoma glándulas } \\
\text { hepatoides }\end{array}$ & $1 \mathrm{~T}-2016$ & $78, X Y, \operatorname{del}(1 \mathrm{q}), 13 \mathrm{hq}+$ \\
\hline 8 & Shi tzu* & 16 & $\begin{array}{l}\text { adenocarcinoma mamario } \\
\text { mixto }\end{array}$ & $1 \mathrm{~T}-2016$ & $78, X X, \operatorname{del}(2 q)$ \\
\hline 9 & Schnauzer & 9 & $\begin{array}{l}\text { adenocarcinoma mamario } \\
\text { mixto/ neoplasia cutánea }\end{array}$ & $1 \mathrm{~T}-2016$ & $79, \mathrm{XX},+13 / 78, \mathrm{XX}$ \\
\hline 10 & Labrador & 9 & Adenocarcinoma de hepatoides & $1 \mathrm{~T}-2016$ & $79, X Y,+18$ \\
\hline 11 & Mestiza & 15 & $\begin{array}{l}\text { Adenocarcinoma mamario } \\
\text { mixto }\end{array}$ & $1 \mathrm{~T}-2016$ & 78,XY,9hq+ \\
\hline 12 & Mestiza & 10 & $\begin{array}{l}\text { Adenocarcionma mamario/ } \\
\text { Lipoma/mastocitoma }\end{array}$ & $1 \mathrm{~T}-2016$ & $80, \mathrm{XX},+13,+18,9 \mathrm{hq}^{+}$ \\
\hline 13 & Labrador & 10 & Adenocarcionma mamario & $2 \mathrm{~T}-2016$ & $78, \mathrm{XX}$ \\
\hline 14 & Schnauzer & 8 & Fibroadenoma mamario & $2 \mathrm{~T}-2016$ & $78, \mathrm{XX}, 9 \mathrm{hq}+$ \\
\hline 15 & $\begin{array}{l}\text { Cocker } \\
\text { Spaniel* }\end{array}$ & 9 & $\begin{array}{l}\text { Carcinoma tubular / evolución } \\
\text { a carcinoma mamario } \\
\text { inflamatorio en } 30 \text { días }\end{array}$ & $2 \mathrm{~T}-2016$ & $79, \mathrm{XX},+13,11 \mathrm{hq}-$ \\
\hline 16 & $\begin{array}{l}\text { Golden } \\
\text { Retriever }\end{array}$ & 13 & Adenocarcinoma mamario & $2 \mathrm{~T}-2016$ & $78, \mathrm{XX}$ \\
\hline 17 & Mestizo & 17 & Carcinoma cutáneo & $2 \mathrm{~T}-2016$ & 78,XY,9hq+ \\
\hline 18 & Dálmata & 6 & Hemangiosarcoma & $2 \mathrm{~T}-2016$ & $78, \mathrm{XX}$ \\
\hline 19 & Mestizo & 6 & Rabdomiosarcoma & $3 \mathrm{~T}-2016$ & $78, X Y$ \\
\hline 20 & $\begin{array}{l}\text { Cocker } \\
\text { Spaniel }\end{array}$ & 10 & Adenocarcinoma mamario & $3 \mathrm{~T}-2016$ & $78, \mathrm{XX}$ \\
\hline 21 & $\begin{array}{l}\text { Mestizo } \\
\text { Pittbull* }\end{array}$ & 10 & Melanoma & $3 \mathrm{~T}-2016$ & $80, \mathrm{XY},+13,+18$ \\
\hline 22 & Poodle & 8 & $\begin{array}{l}\text { Adenocarcionoma de glándulas } \\
\text { ceruminosas }\end{array}$ & $4 \mathrm{~T}-2016$ & 78,XY,9hq+ \\
\hline 23 & $\begin{array}{l}\text { Golden } \\
\text { Retriever* }\end{array}$ & 12 & $\begin{array}{l}\text { Linfoma multicéntrico, } \\
\text { diseminación ósea/ } \\
\text { osteosarcoma }\end{array}$ & $4 \mathrm{~T}-2016$ & $78, \mathrm{XX}, \mathrm{t}(12 ; 22)$ \\
\hline 24 & $\begin{array}{l}\text { Cocker } \\
\text { Spaniel }\end{array}$ & 9 & Hemangiosarcoma cutáneo & $4 \mathrm{~T}-2016$ & $78, \mathrm{XY}, 9 \mathrm{hq}^{+}, 2 \mathrm{hq}+$ \\
\hline 25 & $\begin{array}{l}\text { Scottish } \\
\text { Terrier }\end{array}$ & 6 & Sarcoma cutáneo & $4 \mathrm{~T}-2016$ & $78, X Y$ \\
\hline
\end{tabular}

* Fallecidos 
Cuadro 2. Conteo de cromosomas altamente afectados en perros domésticos con neoplasias durante 2015-2016

\begin{tabular}{|c|c|c|c|c|c|c|}
\hline 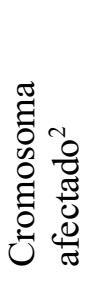 & 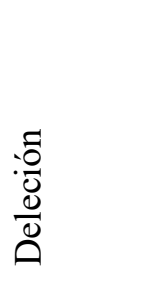 & 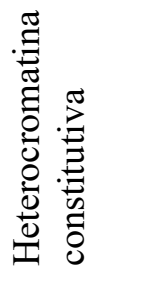 & 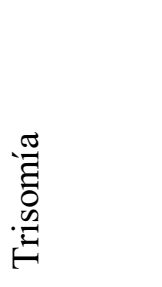 & 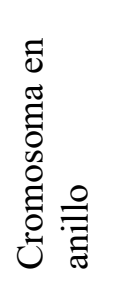 & 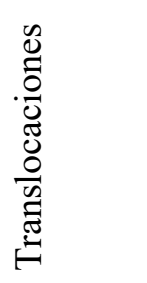 & 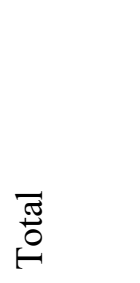 \\
\hline 1 & 1 & & & & & $1(5.6)$ \\
\hline 2 & 1 & 1 & & 1 & & $3(16.8)$ \\
\hline 9 & & 6 & & & & $6(33.4)$ \\
\hline $10^{3}$ & & & & & 1 & $1(5.6)$ \\
\hline 11 & & 1 & & & & $1(5.6)$ \\
\hline $12^{4}$ & & & & & 1 & $1(5.6)$ \\
\hline 13 & & 1 & 7 & & & $8(44.5)$ \\
\hline $18^{3}$ & & & 5 & & 1 & $6(33.4)$ \\
\hline $22^{4}$ & & & & & 1 & $1(5.6)$ \\
\hline 26 & & 1 & 1 & & & $2(11.2)$ \\
\hline Total & $2(11.2)$ & $10(61.1)$ & $13(72.3)$ & $1(5.6)$ & $4(22.4)$ & \\
\hline
\end{tabular}

${ }^{1}$ Porcentajes entre paréntesis

${ }^{2}$ Se incluyen cromosomopatías mixtas y simples

${ }^{3}$ Cromosomas implicados en alteración cromosómica del paciente $\mathrm{N} .{ }^{\circ} 23: 78, \mathrm{XX}, \mathrm{t}(12 ; 22)$

${ }^{4}$ Cromosomas implicados en alteración cromosómica mixta del paciente $\mathrm{N} .{ }^{\circ} 03: 79, \mathrm{XY},+13, \mathrm{t}(10 ; 18)$

La neoplasia mamaria fue la más frecuente afectando más del 70\% de las hembras, principalmente en perros de raza pura y con más de 10 años. Esta elevada prevalencia coincide con la prevalencia de alteraciones citogenéticas reportadas en carcinoma mamario en Argentina (61\%) (Canzoneri et al., 2013). Esta prevalencia señala la importancia y relación con diversos estudios que revelan la presencia de aberraciones cromosómicas conservadas evolutivamente compartidas con la condición neoplásica correspondiente a los seres humanos con patologías linfoproliferativas (Breen y Modiano, 2008; Carvalho et al., 2016). Estas cromosomopatías forman probablemente parte de la inestabilidad genética (Hanahan y Weinberg, 2011).
Los hallazgos más frecuentes fueron las trisomías y las heterocromatinas (Cuadro 2). Las primeras pueden ligarse a una sobreexpresión o malfuncionamiento de los genes contenidos en los cromosomas involucrados; por ejemplo, en el cromosoma 13 donde está contenido el gen c-KiT (ligado a la proliferación celular). Por otro lado, las heterocromatinas constitutivas podrían estar asociadas al silenciamiento de genes reguladores del crecimiento celular, como en el caso del cromosoma 9, afectado frecuentemente, donde, por ejemplo, podría afectarse al gen TIMP2 inhibidor metaloproteinasas, Brac1, entre otros, contribuyendo como uno de los factores desencadenantes de procesos neoplásicos. También se podría interpretar como un cambio epigenético en el genoma 
del paciente (André et al., 1992; Aresu et al., 2011; Reimann-Berg et al., 2012). Asimismo, las deleciones resultan de gran interés, dado que la ausencia de genes controladores de la proliferación, como en el caso de los cromosomas afectados 1 y 2 , podría favorecer el desarrollo neoplásico compatible con los hechos biológicos de la enfermedad (Fleck et al., 2016).

Surge ahora una singular interrogante acerca de las alteraciones cromosómicas múltiples. Todas las alteraciones genéticas celulares promueven en gran medida y a corto plazo el desarrollo de los procesos neoplásicos (Dickinson et al., 2016). Esto implica que los pacientes con alteraciones múltiples están dotándose de una rápida y agresiva evolución que conllevaría a una inestabilidad genética necesaria en los procesos neoplásicos (Thomas et al., 2003; Ujvari et al., 2017). Por otro lado, en el caso de las translocaciones, cuyo producto final constituyen proteínas híbridas ligadas mayormente al aumento de la proliferación en líneas celulares, como el caso de las leucemias en humanos (Thomas et al., 2003; Dickinson et al., 2016; Fleck et al., 2016), donde un perro presentó hiperplasia prostática y otro presentó linfoma multicéntrico, lo cual podría corrobora la familiarización entre los procesos linfoproliferativos humanos y caninos (Ujvari et al., 2017). En el presente estudio, además, se reportó un solo caso de cromosoma en anillo (Cuadro 1), alteración que produciría un aumento en la dificultad del correcto desempeño de los genes contenidos en los cromosomas afectados (Eliasson et al., 1967).

Las hembras fueron las más afectadas, debido principalmente a la alta frecuencia del tumor mamario, resultado que coincide con otros estudios (Mayr y Kramberger-Kaplan, 1992; Dobson, 2013; Borge et al., 2015; Lutful et al., 2016). Sin embargo, la proporción de alteraciones cromosómicas se mantiene elevada (Cuadro 1) entre sexos pese a la pequeña proporción de pacientes machos estudiados. Los pacientes de mejor sobrevida fue- ron los que tenían pocas alteraciones o ninguna, a excepción del carcinoma inflamatorio y fibrosarcoma donde al parecer la malignidad de estas neoplasias es una variable independiente a las alteraciones citogenéticas.

\section{Conclusiones}

Los procesos neoplásicos en caninos domésticos estuvieron acompañados por alteraciones cromosómicas en un porcentaje elevado $(72 \%)$ en la población evaluada que conllevan a inestabilidad génica, necesaria para producirse un proceso neoplásico.

\section{Agradecimientos}

Los autores agradecen la contribución del Lic. OR Ronald Torres-Martínez y del Lic. TM Víctor Rojas-Zumarán por la asesoría estadística y metodológica, respectivamente. A todos los propietarios que permitieron a sus canes contribuir con este estudio que ayudará a complementar la información clínica e individual de cada paciente en el campo de la oncología veterinaria en el Perú.

\section{LitTERATURA CitTAda}

1. André C, Martin E, Cornu F, Hu WX, Wang XP, Galibert F. 1992. Genomic organization of the human c-kit gene: evolution of the receptor tyrosine kinase subclass III. Oncogene 7: 685-691.

2. Aresu L, Giantin M, Morello E, Vascellari $M$, Castagnaro $M$, Lopparelli $R$, et al., 2011. Matrix metalloproteinases and their inhibitors in canine mammary tumors. BMC Vet Res 7: 33. doi: 10.1186/1746-6148-7-33

3. Borge KS, Nord S, Van Loo P, Lingjarde OC, Gunnes G, Alnaes GIG, et al. 2015. Canine mammary tumours are affected by frequent copy number aberrations, including amplification of MYC and loss of PTEN. PLoS One 10(5): e0126371. doi: 10.1371/journal.pone.-0126371 
4. Breen M, Modiano JF. 2008. Evolutionarily conserved cytogenetic changes in hematological malignancies of dogs and humans-man and his best friend share more than companionship. Chromosome Res 16: 145-154. doi: 10.1007/s10577-007-1212-4

5. Breen M. 2008. Canine cytogenetics from band to basepair. Cytogenet Genome Res 120: 50-60. doi: 10.1159/ 000118740

6. Canzoneri R, Rosciani AS, Jorge LC. 2013. Chromosomic breaks and gaps in breast carcinomas of female dogs. Rev Vet 24(2): 81-85.

7. Carvalho MI, Silva-Carvalho R, Pires I, Prada J, Bianchini R, JensenJarolim E, et al. 2016. A comparative approach of tumor-associated inflammation in mammary cancer between humans and dogs. BioMed Res Int 2016: ID 4917387. doi: 10.1155/2016/4917387

8. Czepulkwski B. 2001. Analyzing chromosomes. Oxford: Bios Scientific Publishers. 205 p.

9. Dickinson PJ, York D, Higgins RJ, LeCouteur RA, Joshi N, Bannasch D. 2016. Chromosomal aberrations in canine gliomas define candidate genes and common pathways in dogs and humans. J Neuropathol Exp Neurol 75: 700-710. doi: 10.1093/jnen/nlw042

10. Dobson JM, Lascelles D. 2014. BSAVA Manual of canine and feline oncology. $3^{\text {rd }}$ ed. New Jersey: Wiley \& Sons. $376 \mathrm{p}$.

11. Dobson JM. 2013. Breed-predispositions to cancer in pedigree dogs. ISRN Vet Sci 2013: ID 941275. doi: 10.1155/2013/941275

12. Eliasson K, Gustavsson I, Hulten M, Lindsten J. 1967. The meiotic chromosome of the male dog (Canis familiaris). Hereditas 58: 135-137. doi: 10.1111/j.1601-5223.1967.tb02147.x

13. Fleck K, Erhardt G, Lühken G. 2016. From single nucleotide substitutions up to chromosomal deletions: genetic pause of leucism-associated disorders in ani- mals. Berl Munch Tierarztl Wochenschr 129:269-281.

14. Hanahan D, Weinberg RA. 2011. Hallmarks of cancer: the next generation. Cell 144: 646-674. doi: 10.1016/j.cell.2011.02.013

15. Iannuzzi L, Di Berardino D. 2008. Tools of the trade: diagnostics and research in domestic animal cytogenetics. J Appl Genet 49: 357-366. doi: 10.1007/BF03195634

16. Lutful LF, Alvarez CE, Bird RC. 2016. Canine mammary carcinomas: a comparative analysis of altered gene expression. Vet Sci 3(1): 1. doi: 10.3390/ vetsci3010001

17. Maeda J, Yurkon CR, Fujisawa H, Kaneko M, Genet SC, Roybal EJ, et al. 2012. Genomic instability and telomere fusion of canine osteosarcoma cells. PLoS One 7(8): e43355. doi: 10.1371/journal.pone.0043355

18. Mayr B, Kramberger-Kaplan E, Loupal G, Schleger W. 1992. Analysis of complex cytogenetic alterations in three canine mammary sarcomas. Res Vet Sci 53: 205-211. doi: 10.1016/00345288(92)90111-E

19. Reimann-Berg N, Murua Escobar H, Nolte I. 2012. Relevance of chromosome 13 aberrations in canine tumours. Tierarztl Prax K H 40: 267-270.

20. Seabright M. 1971. A rapid technique for human chromosomes. Lancet 2: 971972.

21. Switonski M, Reimann N, Bosma AA, Long S, Bartnitzke S, Pienkowska A, et al. 1996. Report on the progress of standardization of the G-banded canine (Canis familiaris) karyotype. Chromosome Res 4: 306-309.

22. Thomas R, Smith KC, Ostrander EA, Galibert F, Breen M. 2003. Chromosome aberrations in canine multicentric lymphomas detected with comparative genomic hybridisation and a panel of single locus probes. $\mathrm{Br} \mathrm{J}$ Cancer 89: 1530-1537. doi: 10.1038/ sj.bjc. 6601275 
23. Turner KM, Deshpande V, Beyter D, Koga T, Rusert J, Lee C, Li B, et al. 2017. Extrachromosomal oncogene amplification drives tumour evolution and genetic heterogeneity. Nature 543: 122125. doi: 10.1038 /nature 21356

24. Ujvari B, Roche E, Thomas F. 2017. Ecology and evolution of cancer. Oxford: Elsevier. $290 \mathrm{p}$.

25. Vail D, MacEwen E. 2000. Spontaneously occurring tumors of companion animals as models for human cancer.
Cancer Invest 18: 781-792. doi: 10.3109/ 07357900009012210

26. Verano-Zelada M, Moya-Salazar J, Vega-Vera R. 2016. Cytogenetic analysis in Canis lupus familiaris with neoplasm. Rev Peru Divulg Cient Genet Biol Mol 1(1): 6-9. doi: 10.6084/ m9.figshare.2375371.v3

27. Wang HC, Fedoroff S. 1972. Banding in human chromosomes treated with trypsin. Nature New Bio1 235: 52-54. doi:10.1038/newbio235052a0 